\title{
Prevalencia de enteroparásitos en cachorros comercializados en Puno, Perú
}

\author{
Prevalence of intestinal parasites in puppies marketed in Puno, Peru \\ Carlos Enríquez Añamuro ${ }^{1,4}$, Raquel Watanabe Watanabe ${ }^{2,3}$, \\ Feliciana Vilca de Díaz ${ }^{1}$, Francisco Suárez Aranda ${ }^{3}$
}

\section{Resumen}

El objetivo del presente estudio fue determinar la prevalencia de parásitos intestinales en cachorros comercializados en la ciudad de Puno y evaluar la posible asociación de las variables grupo racial y tipo de comercialización (formal, informal) con la presentación de parasitismo intestinal. Se tomaron muestras fecales de 172 cachorros entre 1 y 4 meses de edad, sin distinción de sexo y grupo racial, del mercado informal, así como de veterinarias y tiendas de mascotas (formal). Las muestras fueron procesadas por las técnicas de examen directo y de concentración por flotación con solución de sulfato de zinc al $33 \%$ (GE 1.18). De las 172 muestras, 90 evidenciaron formas parasitarias (huevos, quistes $\mathrm{u}$ ooquistes), representando una prevalencia de $52.3 \%$. Las prevalencias de parásitos intestinales en cachorros de raza pura y mestizos fueron de 44.2 y $60.5 \%$, respectivamente, y de cachorros del comercio informal y formal fueron de 66.3 y $38.4 \%$, respectivamente. Las especies encontradas fueron Dipylidium caninum, Ancylostoma caninum, Toxascaris leonina, Toxocara canis, Trichuris spp, Giardia spp, Isospora spp y Sarcocystis spp. Se observaron 11 casos de bi-parasitismo, uno de tri-parasitismo y uno de tetra-parasitismo. El tipo de comercialización estuvo asociado a la presencia de parasitismo intestinal $(\mathrm{p}<0.05)$, mas no el grupo racial.

Palabras clave: parasitismo intestinal; prevalencia; cachorros; comercialización; Puno

\section{Abstract}

The aim of this study was to determine the prevalence of intestinal parasites in puppies marketed in the city of Puno and to evaluate the possible association of the breed type and type of commercialization (formal, informal) with the presentation of

${ }^{1}$ Laboratorio de Parasitología Veterinaria, Facultad de Medicina Veterinaria y Zootecnia, Universidad Nacional del Altiplano, Puno, Perú

${ }^{2}$ Carrera de Medicina Veterinaria y Zootecnia, Facultad de Ciencias Veterinarias y Biológicas, Universidad Cientifica del Sur, Lima, Perú

${ }^{3}$ Facultad de Medicina Veterinaria, Universidad Nacional Mayor de San Marcos, Lima, Perú

${ }^{4}$ E-mail: carlitosenriquez@outlook.es

Recibido: 7 de marzo de 2018

Aceptado para publicación: 19 de octubre de 2018 
intestinal parasitism. Faecal samples were taken from 172 puppies, without distinction of sex and breed type, between 1 and 4 months of age, from the informal market, as well as from veterinary centres and pet stores (formal). Samples were processed by direct examination and the flotation concentration technique with $33 \%$ zinc sulphate solution (GE 1.18). Of the 172 samples, 90 showed one or more eggs, cysts or oocysts of parasites, representing a prevalence of $52.3 \%$. The prevalence of intestinal parasites in purebred and mestizo puppies were 44.2 and $60.5 \%$, respectively, and of puppies from informal and formal trade were 66.3 and $38.4 \%$ respectively. The species found were Dipylidium caninum, Ancylostoma caninum, Toxascaris leonina, Toxocara canis, Trichuris spp, Giardia spp, Isospora spp and Sarcocystis spp. There were 11 cases of bi-parasitism, one of triparasitism and one of tetra-parasitism. The type of commercialization was associated with the presence of intestinal parasitism $(\mathrm{p}<0.05)$, but not with the breed.

Key words: intestinal parasitism; prevalence; puppies; commercial trade; Puno

\section{INTRODUCCIÓN}

La tenencia de mascotas en hogares de las ciudades se ha convertido en una práctica frecuente, especialmente, la tenencia de perros y gatos, lo que ha conllevado al incremento del comercio formal e informal. De los animales domésticos, el perro es el más común y usualmente está infectado con parásitos intestinales que no solo deterioran su salud, sino que algunos tienen implicancia zoonótica al comprometer la salud humana.

Los helmintos y los protozoarios son los parásitos intestinales que infectan con mayor frecuencia a los perros. La presencia mórbida de los helmintos puede originar obstrucción intestinal (Rojas-Sereno et al., 2016) y vólvulo intestinal, patologías que comprometen la vida del animal (Soulsby, 1987; Leguía, 2002). Entre los helmintos intestinales que afectan a los caninos se encuentran Ancylostoma caninum, Trichuris vulpis, Strongyloides stercolaris, Dipylidium caninum y Toxocara canis, mientras que, entre los protozoos se encuentran Giardia spp, Trichomonas spp, Balantidium coli, Entamoeba spp y coccidias como Isospora spp, Hammondia spp, Sarcocystis spp, Neospora spp, y Toxoplasma spp (SierraCifuentes et al., 2015). También se ha reportado el hallazgo de parásitos de los géneros
Cryptosporidium y Cyclospora, organismos usualmente asociados con seres humanos inmunosuprimidos (Valerio et al., 2010).

El parasitismo intestinal en los perros tiene implicancia en salud pública debido a que contribuye a la contaminación del medio ambiente con huevos (Canese et al., 2003; Andresiuk et al., 2004; Olivares et al., 2014; Vélez-Hernández et al., 2014) al ser hospederos definitivos de parásitos zoonóticos como Toxocara canis, Ancylostomatidae y Giardia duodenalis (Barriga, 2002). Una hembra adulta de Toxocara canis puede producir entre 25000 y 85000 huevos por día (Glickman y Schantz, 1981). Factores como las tasas reproductivas de los parásitos intestinales y la resistencia de los huevos embrionados a condiciones ambientales adversas y desinfectantes de uso convencional favorecen su supervivencia y proliferación (Diez-Baños et al., 1999). Más aún, la falta de sensibilización acerca de las zoonosis parasitarias, así como la carencia de tratamiento profiláctico adecuado para evitar el parasitismo intestinal aumenta el riesgo de exposición e infección de los animales domésticos y los seres humanos (Blagburn, 2008).

La ingestión accidental de huevos de $T$. canis produce la toxocariasis humana, siendo los niños los más vulnerables debido al contacto directo con las mascotas. La mi- 
gración larvaria de $T$. canis puede, eventualmente, producir los síndromes clínicos denominados larva migrans visceral (LMV) y larva migrans ocular (LMO) (Radman et al., 2006).

La infección transplacentaria por $T$. canis puede causar mortalidad en neonatos debido al gran número de larvas adquiridas en el útero, las que cruzan los alvéolos en su ruta hacia el intestino delgado (Overgaauw y van Knapen, 2013). Los cachorros con infecciones graves pueden expulsar gusanos vivos en el vómito y materia fecal, lo que en muchos casos genera reacciones de preocupación o rechazo hacia la mascota por parte de algunos dueños, ya que la fase adulta de T. canis presenta una longitud relativamente grande en comparación con otros nematodos (Rojas, 2004).

Diversos estudios han sido realizados para estimar las prevalencias de parásitos intestinales en perros en diversas ciudades del Perú y del extranjero. En la ciudad de Lima se ha reportado la presencia de Giardia canis, Isospora spp, Toxocara canis, Dipylidium caninum, Ancylostoma caninum, Cryptosporidium spp, ooquistes de Sarcocystis, Toxascaris leonina y Trichuris vulpis (Serrano-Martínez et al., 2014), patógenos que se muestran en asociaciones de biparasitismo (Trillo-Altamirano et al., 2003; Serrano-Martínez et al., 2014) y triparasitismo (Trillo-Altamirano et al., 2003; Vega et al., 2014). Entre las múltiples especies reportadas en cahorros se encuentran varias con potencial zoonótico: Toxascaris leonina, Ancylostoma caninum, Toxocara canis y Dipylidium caninum (Casasbuenas, 2005).

Los cachorros comercializados informalmente son confinados en forma grupal en ambientes pequeños y antihigiénicos, con presencia de materia fecal, lo que sugiere un factor de riesgo para infecciones parasitarias, virales y bacterianas. Esta situación, lamentablemente, constituye una realidad en las grandes ciudades como Puno, en la que se suma la falta de concienciación de la población respecto al cuidado, protección y bienestar animal. No obstante, se ha visto un mayor interés en la población puneña en cuanto a la tenencia responsable de mascotas, aunque mínima, desde la promulgación de la Ley 30407, Ley de Protección y Bienestar Animal en el Perú (Vega y Watanabe, 2016).

Si bien se han desarrollado numerosas investigaciones sobre detección de parásitos intestinales en caninos en Lima y otras ciudades del Perú, son pocos los trabajos realizados en la ciudad de Puno. El objetivo del presente estudio fue determinar la prevalencia de enteroparásitos en cachorros (Canis lupus familiaris) de la ciudad de Puno y evaluar si la presencia de parasitismo intestinal está asociada a la raza y al tipo de comercialización.

\section{Materiales Y Métodos}

Se colectaron muestras fecales sólidas y semisólidas de 172 cachorros aparentemente sanos, de 1 a 4 meses de edad, sin distinción de raza o sexo, provenientes de cuatro veterinarias (comercio formal) y de 18 comerciantes pertenecientes a la Asociación de Comerciantes de Mascotas de la Feria Sabatina (comercio informal) de la ciudad de Puno, Perú.

Los cachorros fueron muestreados en su punto de venta o domicilio del comerciante entre diciembre de 2011 a febrero de 2012. Las heces fueron colectadas en su totalidad, inmediatamente de ser excretadas sobre un cartón, y colocadas en recipientes rotulados de plástico tapa rosca dentro de una caja con geles refrigerantes hasta su procesamiento en el laboratorio.

Las muestras de heces fueron procesadas en el Laboratorio de Parasitología de la Facultad de Medicina Veterinaria y Zootecnia de la Universidad Nacional del Altiplano (UNAP), Puno. Las muestras fueron 
primeramente examinadas visualmente a fin de descartar la presencia de parásitos adultos o proglótidos de tenia (Truant et al., 1981). Las muestras fueron seguidamente procesadas mediante la técnica de concentración por flotación, utilizando 2-4 g de muestra fecal y sulfato de zinc 33\% (GE 1.18) (Ettinger, 2006). Los parásitos hallados fueron registrados e identificados según su morfología (Zajac y Conboy, 2012).

La comparación estadística de las tasas de prevalencia fue realizada entre grupos raciales (mestizo, de raza) y tipo de comercialización (formal e informal), mediante tablas de contingencia y prueba de Chi cuadrado de Pearson, utilizando el programa estadístico STATA v. 12.1. El tamaño de muestra para este estudio se obtuvo utilizando la fórmula de proporciones para determinar la prevalencia de una enfermedad en una población infinita.

\section{Resultados}

La prevalencia de enteroparásitos en cachorros comercializados en la ciudad de Puno fue de 52.3\% (90/172). El 60.5\% (52/ 86) de cachorros mestizos y $44.2 \%(38 / 86)$ de cachorros de raza estuvieron infectados. Así mismo, el 38.4\% (33/86) de cachorros del comercio formal y el $66.3 \%(57 / 86)$ del comercio informal estuvo infectado con algún tipo de parásito intestinal (Cuadro 1).
El análisis microscópico reveló la presencia de huevos de las especies Ancylostoma caninum, Toxascaris leonina, Toxocara canis, Trichuris vulpis, Giardia spp, Isospora spp y Sarcocystis spp, así como cápsulas ovígeras de Dipylidium caninum, siendo las especies más prevalentes $T$. canis e Isospora spp (Figura 1). Se detectaron helmintos en el $46.5 \%$ de los cachorros $(80 / 172)$ y protozoarios en el $16.9 \%(29 / 172)$ (Cuadro 2). Se presentaron 11 casos de biparasitismo, un tri-parasitismo y un tetra-parasitismo (Cuadro 3).

\section{Discusión}

El comercio informal de mascotas en la ciudad de Puno comprende la venta de un elevado número de crías de perros por año, sumado a la escasez de educación y preocupación de los dueños por la salud de sus mascotas en asuntos básicos como calendario de vacunaciones y desparasitaciones. Lamentablemente, como ocurre en muchas ciudades del Perú, la falta de preocupación de las autoridades municipales o regionales para desarrollar campañas de concienciación sobre protección y bienestar animal, sumado a la carencia de una educación sólida sanitaria en la población humana, siguen siendo factores que favorecen la presencia de parasitismo en animales y humanos.

La importancia de la ejecución de proyectos de investigación o estudios de-

Cuadro 1. Prevalencia de enteroparásitos en cachorros comercializados en la ciudad de Puno, según grupo racial y tipo de comercialización (2011-2012)

\begin{tabular}{llcccc}
\hline \multirow{2}{*}{ Variable } & \multirow{2}{*}{$\mathrm{n}$} & \multicolumn{3}{c}{ Prevalencia } \\
\cline { 4 - 6 } & & 38 & 44.2 & 3.8 & $40.4-48.0^{\mathrm{a}}$ \\
\hline \multirow{2}{*}{ Grupo racial } & De raza & 38 & 60.5 & 3.7 & $56.8-64.2^{\mathrm{b}}$ \\
& Mestizo & 52 & 38.4 & 3.7 & $34.7-42.1^{\mathrm{a}}$ \\
& Fomercio & 33 & 66.3 & 3.6 & $62.7-69.9^{\mathrm{b}}$ \\
\hline Total & Informal & 57 & & & \\
\hline
\end{tabular}


Cuadro 2. Prevalencia de parásitos intestinales en perros cachorros comercializados en la ciudad de Puno (2011-2012)

\begin{tabular}{lcccc}
\hline \multirow{2}{*}{ Parásito intestinal } & \multirow{3}{*}{ Perros (n) } & \multicolumn{3}{c}{ Prevalencia } \\
\cline { 3 - 5 } & & $\%$ & D.E. & Rango \\
\hline Helminto & 57 & 33.1 & 3.6 & $29.5-36.7^{\mathrm{a}}$ \\
$\quad$ Toxocara canis & 7 & 4.1 & 1.5 & $2.6-5.6^{\mathrm{b}}$ \\
Ancylostoma caninum & 6 & 3.5 & 1.4 & $2.1-4.9^{\mathrm{b}}$ \\
$\quad$ Toxascaris leonina & 2 & 1.2 & 0.8 & $0.4-2.0^{\mathrm{c}}$ \\
Trichuris vulpis & 1 & 0.6 & 0.6 & $0-1.2^{\mathrm{c}}$ \\
$\quad$ Capillaria spp & 7 & 4.1 & 1.5 & $2.6-5.6^{\mathrm{b}}$ \\
$\quad$ Dipylidium caninum & & & & \\
Protozoario & 25 & 14.5 & 2.7 & $11.8-17.2^{\mathrm{d}}$ \\
$\quad$ Isospora spp & 3 & 1.7 & 1 & $0.7-2.7^{\mathrm{b}, \mathrm{c}}$ \\
$\quad$ Giardia spp & 1 & 0.6 & 0.6 & $0-1.2^{\mathrm{c}}$ \\
$\quad$ Sarcocystis spp & 172 & 52.3 & & \\
\hline Total de muestras & &
\end{tabular}

Cuadro 3. Parasitismo intestinal múltiple en perros cachorros de la ciudad de Puno (20112012)

\begin{tabular}{llc}
\hline & Parásitos & Casos (n) \\
\hline Biparasitismo & Dipylidium caninum + Isospora spp & 1 \\
& Dipylidium caninum + Toxocara canis & 1 \\
& Giardia spp + Isospora spp & 2 \\
& Ancylostoma caninum + Isospora spp & 1 \\
& Toxocara canis + Isospora spp & 3 \\
& Toxocara canis + Toxascaris leonina & 1 \\
& Ancylostoma caninum + Toxocara canis & 2 \\
Triparasitismo & Ancylostoma caninum + Giardia spp + Isospora spp & 1 \\
Tetraparasitismo & Toxocara canis + Giardia spp + Isospora spp & 1 \\
\hline
\end{tabular}

muestreo para estimar prevalencia de parásitos en caninos no sólo involucra al área de Sanidad Animal, sino también, de Salud Pública. Más aún, la política de intervención de las autoridades sanitarias y profesionales de las ciencias de la salud se resume en el concepto 'One Health' (Una Salud), que hace énfasis en la necesidad de interrelacionar a los profesionales de ciencias de la salud y ciencias agropecuarias en el compromiso de velar por el cuidado de la salud y bienestar de los animales, los seres humanos y del medio ambiente (WHO, 2017). 


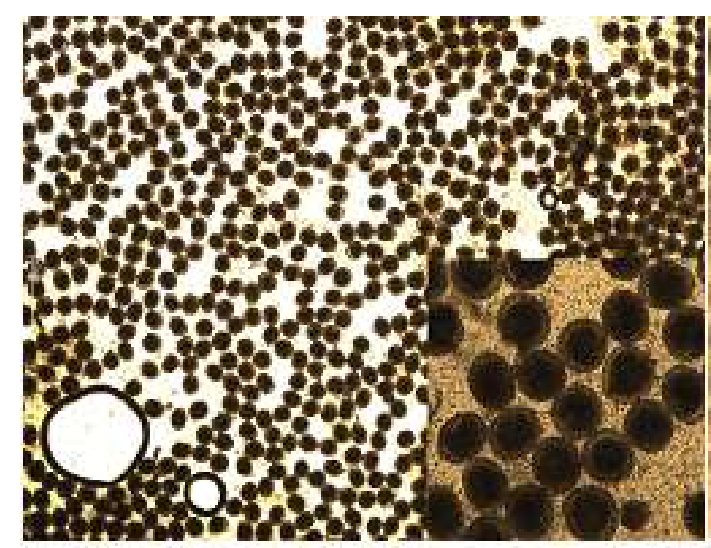

(a)

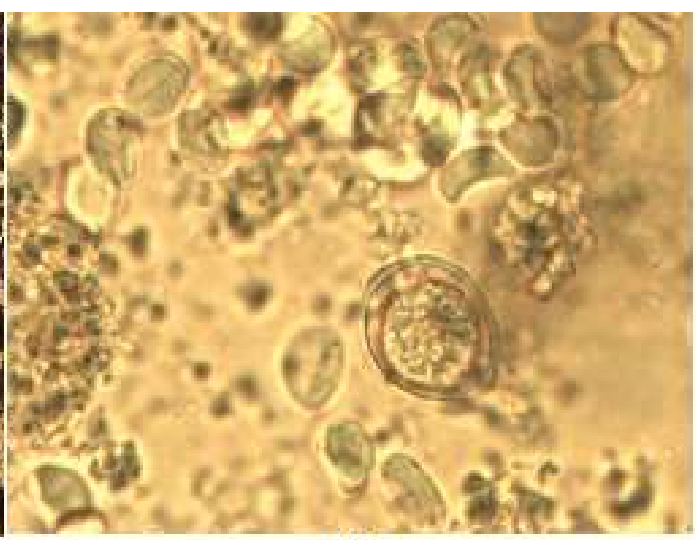

(b)

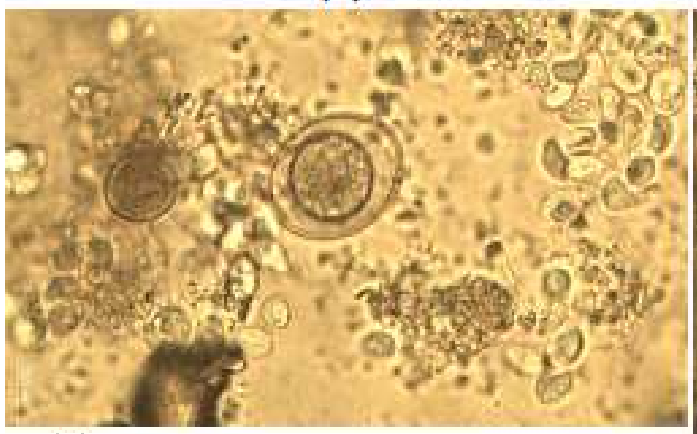

(c)
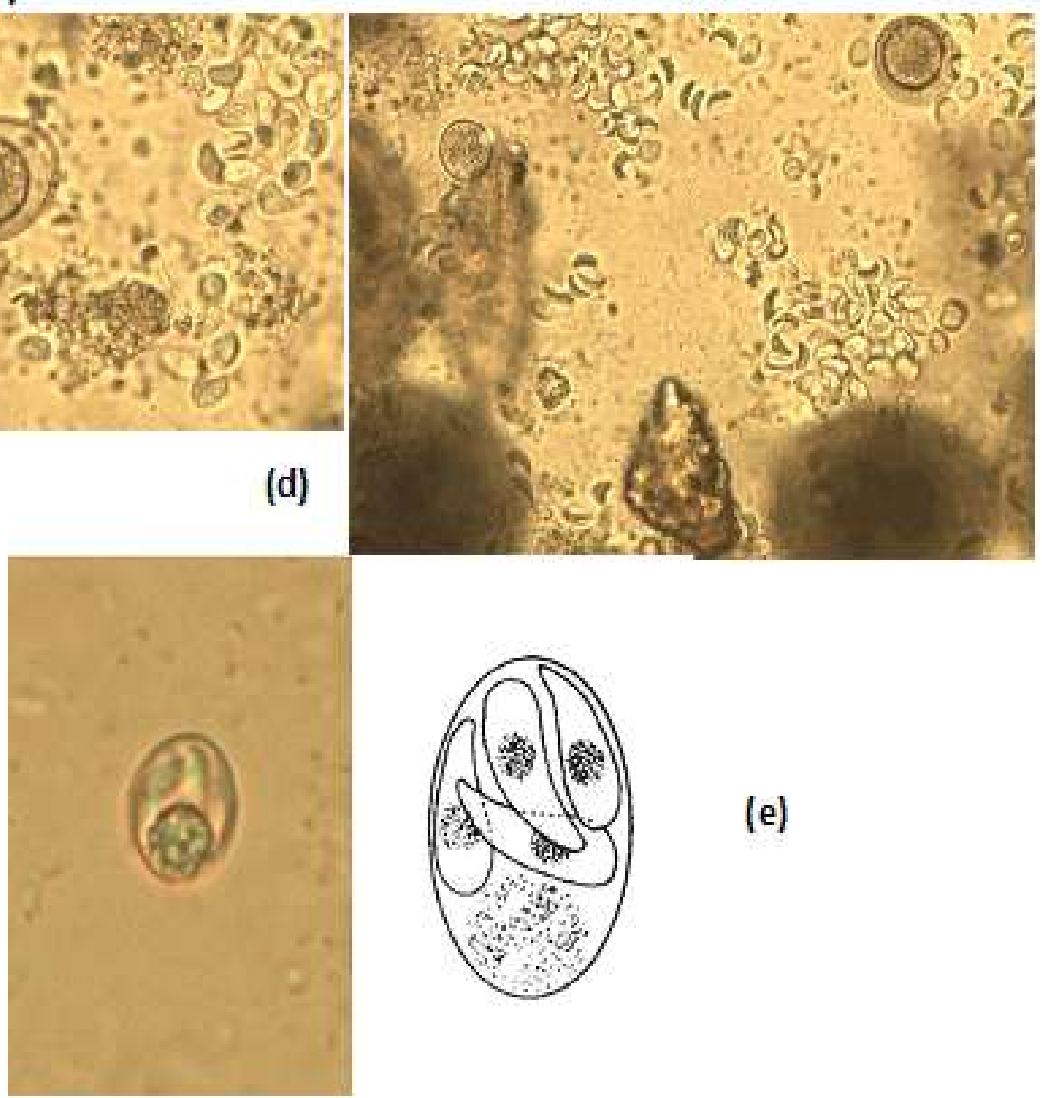

(e)

Figura 1. Huevos de parásitos intestinales de perros de 1-4 meses de edad de la ciudad de Puno (2011-2012). (a) Huevos de Toxocara canis (40x). Vista a 100x en esquina inferior derecha. (b) Quistes de Giardia (examen flotación fecal con sulfato de zinc) e Isospora spp. 400x. (c) Triparasitismo: Quistes de Giardia spp, Isospora spp. 400x. (d) Tetraparasitismo: huevos de Toxocara canis, Giardia spp, Isospora spp. 400x. (e) Sarcocystis spp 
En este estudio se observó una mayor tasa de parasitismo en los perros mestizos $(60.5 \%)$, similar al $76.7 \%$ reportado por Sierra-Cifuentes et al. (2015) en perros de Medellín, Colombia. Contrariamente, Vega et al. (2014) reportaron $82.5 \%$ de parasitosis en caninos de raza pura. No obstante, en el presente estudio, pese a la diferencia de proporciones en animales afectados según el grupo racial, no se evidenció asociación significativa con el parasitismo intestinal.

El tipo de comercialización informal es realizado por personas de medianos a bajos recursos económicos y generalmente está dirigido a personas del mismo estrato económico, quienes difícilmente destinan recursos para la visita veterinaria y cuidados básicos como desparasitación de sus mascotas, o carecen del conocimiento necesario sobre las enfermedades parasitarias. Estos factores culturales y socioeconómicos son importantes factores de riesgo asociados a las infecciones parasitarias (Tortolero et al., 2008). En este estudio, los cachorros comercializados de manera informal presentaron mayor parasitismo intestinal respecto a aquellos comercializados formalmente $(\mathrm{p}<0.05)$.

Las tasas de monoparasitismo fueron las más frecuentes, lo que concuerda con estudios previos (Leguía et al., 1980; Dávalos et al., 2000; Trillo-Altamirano et al., 2003; Tortolero et al., 2008; Vilca y Anccasi, 2013; Vega et al., 2014), siendo T. canis el parásito con la prevalencia más alta seguido de $I$. canis. La alta prevalencia de $T$. canis puede deberse a la mayor probabilidad de transmisión vertical del parásito (Leguía, 2002; Vega et al., 2014).

La presencia de Ancylostoma caninum, Toxascaris leonina, Trichuris vulpis, Capillaria spp, Dipylidium caninum, Giardia spp, Isospora ohioenis y Sarcocystis spp coinciden con las reportadas en estudios previos realizados en distintas ciudades del Perú. Iannacone et al. (2001) reportó una prevalencia de $33.3 \%$ de $D$. caninum, seguido de $T$. canis (16.6\%) y $T$. pisiformis (10\%) en perros de San Juan de Lurigancho, Lima. Por otro lado, Dunois y Vaca (2005) determinaron una mayor proporción de infecciones por Toxocara $\mathrm{sp}$ (60.79\%), seguido de Ancylostoma sp (16.6\%) en Santa Cruz de la Sierra, Bolivia, similar a lo encontrado en este estudio.

Se observan grandes diferencias en términos de prevalencia de parasitismo entérico, sean prevalencias globales o por especies, tanto en el Perú como en otras ciudades del extranjero. Estas diferencias son probablemente debidas a diferentes factores culturales, socioeconómicos (Gorman et al., 2006), ambientales (Andresiuk et al., 2007) y métodos de diagnóstico empleados (Truant et al., 1981; Mandarino-Pereira et al., 2010).

En cuanto al parasitismo múltiple, la asociación de T. canis e I. canis fue la más frecuente, hecho que puede ser explicado por las altas prevalencias encontradas de ambos parásitos. Este hallazgo coincide con lo reportado por Vega et al. (2014), en cuyo estudio se encontró frecuencias de 88 y $99 \%$ para T. canis e I. canis, respectivamente. Así mismo, Trillo-Altamirano et al. (2003) observaron $7.7 \%$ de bi-parasitismo de $T$. canis y $D$. caninum explicable por la elevada producción de huevos de $T$. canis y su resistencia a temperaturas extremas y compuestos químicos que le confiere mayor capacidad de diseminación en el suelo, y a la infestación masiva con pulgas que son los hospederos intermediarios de D. caninum. Por otro lado, se ha reportado $12.4 \%$ de tri-parasitismo de T. canis - I. canis - T. leonina en cachorros caninos vendidos en el Cercado de Lima (Vega et al., 2014), a diferencia del presente estudio donde solo se observó tri-parasitismo de A. caninum - Giardia spp - Isospora spp.

Multi-parasitismo en perros ha sido reportado en Corrientes (Milano y Oscherov, 2002) y Mar del Plata, Argentina (Andresiuk et al., 2004), Río Grande del Sur, Brasil (Hoffmann et al., 2000), Santa Clara, Cuba (Artiles et al., 2012), Islas Galápagos, Ecua- 
dor (Gingrich et al., 2010), Quindío, Colombia (Giraldo et al., 2005), Vela, Venezuela (Tortolero et al., 2008), Escárcega, México (Encalada-Mena et al., 2011), Seropédica, Brasil (Mandarino-Pereira et al., 2010) y Querétaro, México (Fernández y Cantó, 2002), involucrando una gran variedad de helmintos y protozoarios.

La alta prevalencia de T. canis en la población canina supone también una mayor exposición en la población humana. En Lima, se han reportado seroprevalencias de 23 y $30 \%$ en humanos (Espinoza et al., $2003 \mathrm{y}$ Alarcón et al., 2010, respectivamente), siendo la mayoría pacientes asintomáticos. Similares seroprevalencias han sido reportadas en Argentina y Brasil, especialmente en niños (Moreira et al., 1998; Alonso et al., 2000; Radman et al., 2000). Los factores de riesgo asociados con toxocariosis humana son la presencia de perros en los hogares (Alarcón et al., 2010), especialmente en viviendas con pisos de tierra (Canese et al., 1999).

\section{Conclusiones}

- La prevalencia de enteroparásitos en perros cachorros (Canis lupus familiaris) comercializados en la ciudad de Puno fue de 52.3\% (90/172).

- El tipo de comercialización de los cachorros (formal vs. informal) estuvo asociado a la presencia de parasitismo intestinal, mas no el grupo racial.

- Las especies encontradas fueron Dipylidium caninum, Ancylostoma caninum, Toxascaris leonina, Toxocara canis, Trichuris spp, Giardia spp, Isospora spp y Sarcocystis spp.

\section{Literatura Citada}

1. Alarcón M, Iannacone J, Espinoza Y. 2010. Parasitosis intestinal, factores de riesgo y seroprevalencia de toxocariosis en poblaciones del parque industrial de Huaycán, Lima, Perú. Neotrop Helminthol 4: 17-36.

2. Alonso JM, Bojanich MVI, Chamorro M, Gorodner JO. 2000. Toxocara seroprevalence in children from a subtropical city in Argentina. Rev Inst Med Trop Sp 42: 235-237. doi: 10.1590/ S0036-46652000000400010

3. Andresiuk V, Sardella N, Denegri G 2007. Seasonal fluctuations in prevalence of dog intestinal parasites in public squares of Mar del Plata city, Argentina and its risk for humans. Rev Argent Microbiol 39: 221-224.

4. Andresiuk MV, Rodríguez, Fenegri GM, Sardella NH, Hollmann P. 2004. Relevamiento de parásitos zoonóticos en materia fecal canina y su importancia para la salud de los niños. Arch Argent Pediatr 102: 325-329.

5. Artiles E, Ruiz L, Rodríguez L, Hernández Y. 2012. Contaminación por heces de caninos en calles de Santa Clara: un riesgo potencial para la transmisión de enfermedades parasitarias zoonóticas. REDVET 13(6). [Internet]. Disponible en: http://www.redalyc.org/ pdf/636/63624434009.pdf

6. Barriga O. 2002. Las enfermedades parasitarias de los animales domésticos. Costa Rica: Ed Germinal. 247 p.

7. Blagburn BL. 2008. Parasitic diseases you should know about (Proceedings). [Internet]. Disponible en: http:// veterinarycalendar.dvm $360 . \mathrm{com} /$ parasitic-diseases-you-should-knowabout-proceedings

8. Canese A, Domínguez $R$, Otto $C$, Ocampos C, Mendonca E. 2003. Huevos infectivos de Toxocara en arenas de plazas y parques de Asunción, Paraguay. Rev Chilena Pediatría 74: 611-616. doi: $10.4067 / \mathrm{S} 0370-41062003000600010$

9. Canese A, Orué M, Paciello L, Rodríguez H. 1999. Huevos infectivos de Toxocara en el suelo de la ciudad de Asunción, Paraguay. Rev Paraguaya Microbiol 19: 42-48. 
10. Casasbuenas P. 2005. Infección por Dipylidium caninum. Rev Colomb Gastroenterol 20: 86-89.

11. Dávalos M, Pachas O, Pérez V. 2000. Toxocariosis en Canis familiaris y suelo en el distrito de Chincha alta (19981999). En: IV Congreso Peruano de Parasitología. Lima, Perú.

12. Diez-Baños P, Diez-Baños $N$, Morrondo M. 1999. Nematodosis: toxocariosis, toxocascaridosis, ancilostomatidosis, tricuriosis, estrongiloidosis, espirocercosis y olulanosis. En: Cordero del Campillo M (ed). Parasitología veterinaria. Madird: McGraw-Hill Interamericana. p 637-651.

13. Dunois UT, Vaca RJL. 2005. Prevalencia de nematodes gastrointestinales en canes de la ciudad de Cochabamba. Tesis de Médico Veterinario Zootecnista. Santa Cruz de la Sierra: Univ. Autónoma Gabriel Rene Moreno. 45 p.

14. Encalada-Mena LA, Duarte-Ubaldo EI, Vargaz-Magaña JJ, GarcíaRamírez MJ, Medina-Hernández RE. 2011. Prevalencia de parásitos gastroentéricos de cánidos en la ciudad de Escárcega, Campeche, México. Universidad y Ciencia 27: 209-217.

15. Espinoza Y, Huapaya P, Sevilla C, Huiza A, Jiménez S, Náquira C. 2003. Toxocariosis humana: seroprevalencia en población de Lima mediante la técnica de ELISA. Anales Facultad de Medicina 64: 228-232. doi: 10.15381/ anales.v64i4.1420

16. Ettinger SJ. 2006. Tratado de medicina interna veterinaria. $5^{\mathrm{a}}$ ed. Buenos Aires: Intermédica. $1181 \mathrm{p}$.

17. Fernández F, Cantó GJ. 2002. Frecuencia de helmintos en intestinos de perros sin dueño sacrificados en la ciudad de Querétaro, Querétaro, México. Vet México 33: 247-253.

18. Gingrich EN, Scorza AV, Clifford EL, Olea-Popelka FJ, Lappin MR. 2010. Intestinal parasites of dogs on the Galapagos Islands. Vet Parasitol 169: 404-407. doi: 10.1016/j.vetpar.2010.01.018
19. Giraldo MI, García NL, Castaño JC. 2005. Prevalencia de helmintos intestinales en caninos del departamento del Quindío. Biomédica 25: 346-352. doi: 10.7705/biomedica.v25i3.1359

20. Glickman LT, Schantz PM. 1981. Epidemiology and pathogenesis of zoonotic toxocariasis. Epidemiol Rev 3: 230-250.

21. Gorman T, Soto A, Alcaino H. 2006. Parasitismo gastrointestinal en perros de comunas de Santiago de diferente nivel socioeconómico. Parasitol Latinoam 61: 126-132. doi: 10.4067/S0717-77122006000200005

22. Hoffmann AN, Beltrao N, Botton Sde A, Caminha BX, de la Rue ML. 2000. Intestinal nematodes of stray dogs as zoonoses agents in D. Pedrito City (RSBrazil). Bol Chileno Parasitol 55: 92-93. doi: 10.4067/S0365-94022000000300011

23. Iannacone JO, Cordova KM, Wong $\boldsymbol{R} \boldsymbol{V}$. 2001. Estructura comunitaria de helmintos de perros vagabundos de San Juan de Lurigancho. Rev Bras Zool 18: 277-288. doi: 10.1590/S0101-81752001000500024 .

24. Leguía G. 2002. Enfermedades parasitarias de perros y gatos. Epidemiología y control $2^{\mathrm{a}}$ ed. Lima: Ed de Mar. $155 \mathrm{p}$.

25. Leguía G, Guerrero C, Arévalo $F$. 1980. Prevalencia de helmintos gastrointestinales en caninos de la provincia de Huancayo. Rev Inv Pec 5: 15-20.

26. Mandarino-Pereira A, de Souza FS, Lopes CW, Pereira MJ. 2010. Prevalence of parasites in soil and dogs feces according to diagnostic tests. Vet Parasitol 170: 176-181. doi: 10.1016/ j.vetpar.2010.02.00

27. Milano F, Oscherov EB. 2002. Contaminación por parásitos caninos de importancia zoonótica en playas de la ciudad de Corrientes, Argentina. Parasitol Latinoam 57: 110-123. doi: 10.4067/ S0717-77122002000300006

28. Moreira S, Leao M, Mendonca H, Pereira F. 1998. Prevalence of antiToxocara antibodies in a random sample of inpatients at a children's hospital in 
Vitoria, Espírito Santo, Brazil. Rev Inst Med Trop Sp 40: 259-261. doi: 10.1590/ S0036-46651998000400010

29. Olivares P, Valenzuela G, Tuemmers C, Parodi J. 2014. Descripción de parásitos presentes en muestras fecales recolectadas en plazas del sector céntrico de la ciudad de Temuco, Chile. Rev Inv Vet Perú 25: 406-413. doi: 10.15381/ rivep.v25i3.10119

30. Overgaauw PA, van Knapen F. 2013. Veterinary and public health aspects of Toxocara spp. Vet Parasitol 193: 398403. doi: 10.1016/j.vetpar.2012.12.035

31. Radman NE, Archelli SM, Fonrouge RD, del V Guardis M, Linzitto OR. 2000. Human toxocarosis. Its seroprevalence in the city of La Plata. Mem I Oswaldo Cruz 95: 281-285. doi: 10.1590/S0074-02762000000300001

32. Radman N, Archelli S, Burgos L, Fonrouge R, del Valle M. 2006. Toxocara canis en caninos. Prevalencia en la ciudad de La Plata. Acta Bioquim Clin L 40: 41-44.

33. Rojas M. 2004. Nosoparasitosis de los rumiantes domésticos peruanos $2^{\mathrm{a}}$ ed. Lima: Martegrad. $146 \mathrm{p}$.

34. Rojas-Sereno ZE, Segura-Monroy VM, Rincón M, Granados J, Brieva C. 2016. Intususcepción ileal generada por Prosthenorchis sp en un tití gris (Saguinus leucopus) mantenido en un centro de recepción de fauna en Colombia. Rev Méd Vet 31: 75-83. doi: 10.19052/mv.3711

35. Serrano-Martínez E, Tantaleán M, Castro V, Quispe M, Casas G. 2014. Estudio retrospectivo de frecuencia de parásitos en muestras fecales en análisis rutinarios de laboratorio. Rev Inv Vet Perú 25: 113-116. doi: 10.15381/rivep.v25i1.8476

36. Sierra-Cifuentes V, Jiménez-Aguilar $J$, Alzate A, Cardona-Arias J, RíosOsorio L. 2015. Prevalencia de parásitos intestinales en perros de dos centros de bienestar animal de Medellín y el oriente antioqueño (Colombia), 2014. Rev Med Vet 30: 55-66.

37. Soulsby E. 1987. Parasitología y enfermedades parasitarias en animales domésticos $7^{\mathrm{a}}$ ed. México DF: Interamericana. $823 \mathrm{p}$.

38. Tortolero LJ, Cazorla DJ, Morales P, Acosta ME. 2008. Prevalencia de enteroparásitos en perros domiciliarios de la ciudad de Vela, estado Falcón, Venezuela. Rev Cient LUZ 18: 312-319.

39. Trillo-Altamirano MP, Carrasco AJ, Cabrera R. 2003. Prevalencia de helmintos enteroparásitos zoonóticos y factores asociados en Canis familiaris en una zona urbana de la ciudad de Ica, Perú. Parasitol Latinoam 58: 136-141. doi: 10.4067/S0717-77122003000300009

40. Truant AL, Elliott SH, Kelly MT, Smith JH. 1981. Comparison of formalin-ethyl ether sedimentation, formalin-ethyl acetate sedimentation, and zinc sulfate flotation techniques for detection of intestinal parasites. J Clin Microbiol 13: 882-884.

41. Valerio I, Ulate R, Soto M, Chinchilla M. 2010. Hallazgo de parásitos oportunistas en perros (Canis familiaris) del área metropolitana de Costa Rica. Rev Ibero-Latinoam Parasitol 69: 52-59.

42. Vega S, Serrano-Martínez E, Grandez R, Pilco M, Quispe M. 2014. Parásitos gastrointestinales en cachorros caninos provenientes de la venta comercial en el Cercado de Lima. Salud Tecnol Vet 2: 71-77. doi: 10.20453/stv.v2i2.2242

43. Vega S, Watanabe R. 2016. Análisis de la Ley 30407 «Ley de Protección y Bienestar Animal» en el Perú. Rev Inv Vet Perú 27: 388-396. doi: 10.15381/ rivep.v27i2.11664

44. Vélez-Hernández L, Reyes-Barrera K, Rojas-Almaráz D, Calderón-Oropeza M, Cruz-Vásquez J, Arcos-García J. 2014. Riesgo potencial de parásitos zoonóticos presentes en heces caninas en Puerto Escondido, Oaxaca. Salud Publica de México 56: 625-630. doi: 10.21149/spm.v56i6.7389 
45. Vilca de Díaz F, Anccasi M. 2013. Enteroparásitos en perros (Canis familiaris) y gatos (Felis catus) de la provincia de Puno. Rev Invest Altoandinas 15: 117-122. doi: 10.18271/ ria.2013.21
46. [WHO] World Health Organization. 2017. One health. [Internet]. Disponible en: http://www.who.int/features/qa/ one-health/en/

47. Zajac AM, Conboy GA. 2012. Veterinary clinical parasitology. $8^{\text {th }}$ ed. New Jersey: Wiley-Blackwell. 368 p. 